\title{
Modeling Bistable Composite Laminates for Piezoelectric Morphing Structures
}

\author{
Darryl V. Murray and Oliver J. Myers \\ Smart Materials Research Laboratory, Department of Mechanical Engineering, Mississippi State University, MS 39762, USA \\ Correspondence should be addressed to Oliver J. Myers; myers@me.msstate.edu
}

Received 22 January 2013; Accepted 17 February 2013

Academic Editors: L. Chang and V. Sglavo

Copyright (C) 2013 D. V. Murray and O. J. Myers. This is an open access article distributed under the Creative Commons Attribution License, which permits unrestricted use, distribution, and reproduction in any medium, provided the original work is properly cited.

\begin{abstract}
A sequential modeling effort for bistable composite laminates for piezoelectric morphing structures is presented. Thin unsymmetric carbon fiber composite laminates are examined for use of morphing structures using piezoelectric actuation. When cooling from the elevated cure temperature to room temperature, these unsymmetric composite laminates will deform. These postcure room temperature deformation shapes can be used as morphing structures. Applying a force to these deformed laminates will cause them to snap through to another shape. This bistability arises from the fabrication process of the thin unsymmetric laminates. The snap through force studied here will be controlled by using piezoelectricity. Macrofiber composite (MFC) actuators are used for piezoelectric actuation. In this research, an analytical modeling method is presented to accurately depict the piezoelectric morphing structures. Sequential numerical modeling of the cure process to account for residual stresses and postcured shapes and piezoelectric morphing structure is done to predict the piezoelectric actuated displacements of the thin unsymmetric composite laminates. Analytical and numerical models are compared to experimental methods and results.
\end{abstract}

\section{Introduction}

Smart material systems are vastly becoming an integral part in engineering applications. One of the phenomena used in smart material systems is piezoelectricity. Working with unsymmetric bistable composites, piezoelectric effects can be implemented to achieve a snap through to the other cylindrical stable shape of the composite. This shape change caused by piezoelectric effects can be coupled with other domains and used as sensor or actuator. The objective of this research was to use piezoelectric effects for actuation. Bistability of the composite laminate is achieved during fabrication. After the cure process, multiple deformation shapes can be observed based on the ply orientation, material, and thickness of the laminate [1]. This deformation is due to the thermal strain gradient between the layers of the laminate and cure shrinkage during the cure process [2]. As an essential part of the smart material morphing system, the cure process and the resulting postcure deformation shapes are heavily investigated. In order to accurately depict piezoelectric morphing structures, the postcure room temperature shapes need to be characterized. This is done by using a Rayleigh-Ritz technique that accounts for the large out-of-plane strains occurring during the cooling of the laminate [3-7]. Piezoelectricity exhibits electromechanic coupling making it very useful for sensing and actuation in smart material systems. Actuation in this research is established by using the converse piezoelectric effect. For the converse piezoelectric effect, an electric field is applied to the piezoelectric crystal causing strain in the crystal [8]. Applying a voltage to the MFC actuator induces this mechanical strain in the patch which is then transferred from the actuator to the composite laminate causing it to snap through to an orthogonal cylindrical shape, cylindrical shape II. With removal of the applied voltage, stable composite laminates will continue to stay in cylindrical shape II while unstable composite laminates will snap back to its original postcure shape. Along with experimentation, analytical and finite element analysis models have been implemented to compare postcure deformation shapes and actuated deformation of the composite laminates. Methods to accurately characterize the piezoelectric effect are given through analytical and numerical models. These models are then compared to experimental results. 


\section{Analytical Models for Morphing Structures}

The MFC actuator used for experimentation is distributed by Smart Material Corporation. The MFC was invented by NASA in 1996 and since then has been continued to be improved for many applications. The piezoelectricity of the MFC actuator is contained in the rectangular piezoceramic rods of the actuator [9]. These rods are sandwiched between electrodes and polyimide film. The electrodes are in an interdigitated pattern. This pattern allows for direct application of the voltage to the rods, which is the converse piezoelectric effect. Once the voltage is applied, the actuator will elongate or deform. This system enables in-plane poling and actuation, which are both used for this research. The operation mode used for experimentation was the $\mathrm{d} 33$ operating mode. This mode elongates the rods when a voltage is applied. The tensor notation for the operation mode can be broken down to better understand the proper applications and differences between other operation modes. The first variable, 3 , represents the axis of polarization for the material. This direction, 3, is through the thickness of the material also known as the $z$ direction if in $x, y, z$ coordinates making the 1 and 2 directions align in the plane of the piezoelectric material. The second variable, 3 , represents the direction of which the state of the piezoelectric material should be analyzed [10]. With the $d_{33}$ operation mode and the assumption of free strain, the constitutive equations are reduced. Free strain is the strain produced in the piezoelectric material when there is no resistive stress on the material. For piezoelectric analysis, the plates or in this case MFC actuators were classified as thin plates. Thin plates are those with a ratio of thickness to length less than $1 / 20$. This allows the complex threedimensional problem to be reduced to a problem in two dimensions [10]. Using the same assumptions of the classical plate theory, the thin plate is now classified as a plane stress problem. The analysis implements the total potential energy approach similar to the postcure shape analytical work. The Ritz method is used for the variational principal solution and is reduced to a set of linearly independent equations by an assumed form of generalized coordinates. The generalized coordinates are then used to represent the potential energy of the system. This understanding of the effects of the cure process is needed to accurately characterize the piezoelectric effects for composite laminate morphing structures. The thermal stresses and strains [3] are calculated to determine the cooling effects on the composite laminates. The middle surface strains and curvatures can then be approximated:

$$
\begin{gathered}
u^{o}=c x-\frac{a^{2} x^{3}}{6}-\frac{a b x y^{2}}{4}, \\
v^{o}=d y-\frac{b^{2} y^{3}}{6}-\frac{a b x^{2} y}{4}, \\
w^{o}=\frac{1}{2}\left(a x^{2}+b y^{2}\right),
\end{gathered}
$$

where $u^{o}, v^{o}$, and $w^{o}$ are the displacement fields; $x, y$, and $z$ are coordinate directions; and $a, b, c$, and $d$ are coefficients to be determined.
The strains $\varepsilon_{x}, \varepsilon_{y}, \gamma_{x y}$ are approximated using

$$
\begin{gathered}
\varepsilon_{x}=\varepsilon_{x}^{o}+z \kappa_{x}^{o}, \\
\varepsilon_{y}=\varepsilon_{y}^{o}+z \kappa_{y}^{o}, \\
\gamma_{x y}=\gamma_{x y}^{o}+z \kappa_{x y}^{o} .
\end{gathered}
$$

The stresses $\sigma_{x}, \sigma_{y}, \sigma_{x y}$ are functions of the reduced stiffness $\left(Q_{i i}\right)$ and thermal stresses and are given by

$$
\begin{gathered}
\sigma_{x}=Q_{11} \varepsilon_{x}+Q_{12} \varepsilon_{y}+Q_{16} \gamma_{x y}-\sigma_{x}^{T}, \\
\sigma_{y}=Q_{12} \varepsilon_{x}+Q_{22} \varepsilon_{y}+Q_{26} \gamma_{x y}-\sigma_{y}^{T}, \\
\sigma_{x y}=Q_{16} \varepsilon_{x}+Q_{26} \varepsilon_{y}+Q_{66} \gamma_{x y}-\sigma_{x y}^{T} .
\end{gathered}
$$

The postcure room temperature shape can then be modeled. This step does not include bonding the MFC actuator; therefore, only thermal effects are accounted for. The strain energy or total potential energy is given by

$$
\begin{array}{r}
\Pi_{1}=\frac{1}{2} \int_{-L_{x} / 2}^{L_{x} / 2} \int_{-L_{y} / 2}^{L_{y} / 2} \int_{z_{0}}^{z_{2}}[ \\
{\left[\left(\sigma_{x}-\sigma_{x}^{T}\right) \varepsilon_{x}+\left(\sigma_{y}-\sigma_{y}^{T}\right) \varepsilon_{y}\right.} \\
\left.+\left(\sigma_{x y}-\sigma_{x y}^{T}\right) \gamma_{x y}\right] d x d y d z .
\end{array}
$$

Carrying out the integrations in (4), the potential energy is then reduced to an algebraic equation in terms of the coefficients $a, b, c$, and $d$. Once the integrations are computed, (4) will show that the potential energy is also in terms of material properties and geometries of the laminate where $L_{i}$ are the lengths of the respective directions. These coefficients are then determined by solving the nonlinear algebraic equations that are reduced from equating to zero the first variation of the potential energy with respect to the coefficients

$$
\frac{\partial \Pi}{\partial C_{i}}=0, \quad i=1, \ldots, 4,
$$

where $C$ is $a, b, c$, and $d$. These solutions relate to the equilibrium shapes of the cooled room temperature laminate. After curing and cooling the laminate, the MFC actuator was bonded to the deformed composite laminates. When using the total potential energy to model the postcure shapes, the driving force was the heat transfer taking place. Now for piezoelectric analysis, the driving force will be piezoelectricity. Similar to (4), the total potential energy in response to the MFC actuation is given by

$$
\begin{aligned}
\Pi_{2}=\frac{1}{2} \int_{-L_{x}^{\mathrm{MFC}} / 2}^{L_{x}^{\mathrm{MFC}} / 2} \int_{-L_{y}^{\mathrm{MFC}} / 2}^{L_{y}^{\mathrm{MFC}} / 2} \int_{z_{2}}^{z_{3}}[ & \left(\sigma_{x}-{ }^{a} \sigma_{x}^{E s}\right) \varepsilon_{x}+\left(\sigma_{y}^{a}-\sigma_{y}^{E s}\right) \varepsilon_{y} \\
& \left.+\left(\sigma_{x y}^{a}-\sigma_{x y}^{E s}\right) \gamma_{x y}\right] d x d y d z
\end{aligned}
$$

where the $\sigma_{x}^{a}, \sigma_{y}^{a}, \sigma_{x y}^{a}, \sigma_{x}^{E s}, \sigma_{y}^{E s}, \sigma_{x y}^{E s}, \varepsilon_{x}, \varepsilon_{y}, \gamma_{x y}$ are the stresses and strains in the MFC actuator and $L_{i}^{\mathrm{MFC}}$ and the directional 


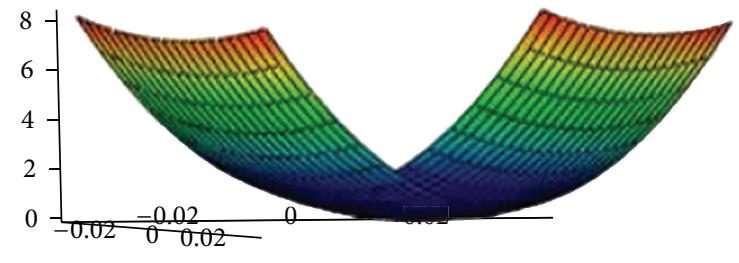

(a) Room temperature shape

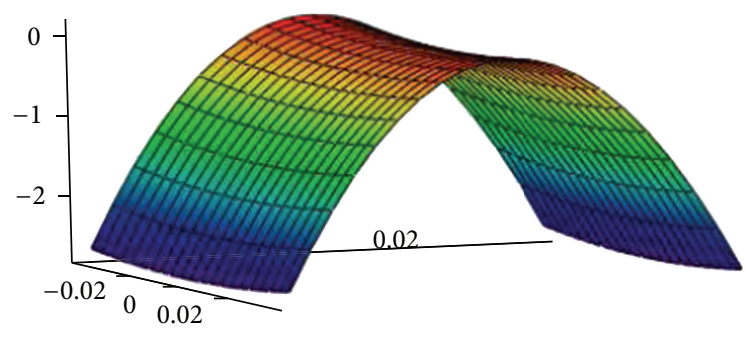

(b) Actuated shape

FIGURE 1: [0/90] room temperature shape versus actuated shape.

lengths of the actuator. The stresses induced by bonding the MFC actuator to the laminate are given by

$$
\begin{gathered}
\sigma_{x}^{a}=Q_{11} \varepsilon_{x}+Q_{12} \varepsilon_{y}+Q_{16} \gamma_{x y}-\sigma_{x}^{E s}, \\
\sigma_{y}^{a}=Q_{12} \varepsilon_{x}+Q_{22} \varepsilon_{y}+Q_{26} \gamma_{x y}-\sigma_{y}^{E s}, \\
\sigma_{x y}^{a}=Q_{16} \varepsilon_{x}+Q_{26} \varepsilon_{y}+Q_{66} \gamma_{x y}-\sigma_{x y}^{E s},
\end{gathered}
$$

which are the stresses in the actuator. The equations for the stresses induced by bonding the MFC actuator are given by:

$$
\begin{gathered}
\sigma_{x}^{E s}=Q_{11} \varepsilon_{x}^{E}+Q_{12} \varepsilon_{y}^{E}+Q_{16} \gamma_{x y}^{E}, \\
\sigma_{x}^{E s}=Q_{11} \varepsilon_{x}^{E}+Q_{12} \varepsilon_{y}^{E}+Q_{16} \gamma_{x y}^{E}, \\
\sigma_{x}^{E s}=Q_{11} \varepsilon_{x}^{E}+Q_{12} \varepsilon_{y}^{E}+Q_{16} \gamma_{x y}^{E} .
\end{gathered}
$$

The strains of the actuator are as follows:

$$
\varepsilon_{x}^{E}=d_{11} \frac{\Delta V}{\Delta x_{1}}
$$

with $V$ being the input voltage and $x_{1}$ the electrode spacing. The total potential energy for the actuation of the laminate, taking the postcure room temperature deformation into account, is then given as

$$
\Pi_{3}=\Pi_{1}+\Pi_{2} \text {. }
$$

Using the same techniques, the undetermined coefficients $a, b, c$, and $d$ can be found by minimizing the total potential energy, $\Pi_{3}$, thus, giving the actuated shapes for the MFC actuator and laminate.

These techniques were used to model the piezoelectric actuation for the $[0 / 90],\left[0_{2} / 90_{2}\right]$, and [0/45] laminates. Figure 1 shows the characterization results of the $[0 / 90]$ laminate. The first mesh in the figure represents the room temperature shape of the [0/90] laminate. The second mesh is the predicted piezoelectric actuated shape.

Analytical models were done for the $\left[0_{2} / 90_{2}\right]$ laminate using the previously described method. With more plies than the [0/90] laminate, the curvature is expected to be different due to the increase of the $90^{\circ}$ plies. When compared to the [0/90] laminate, this curvature is in opposite direction along the other axis. The equilibrium shapes, shown as meshes, of the $\left[0_{2} / 90_{2}\right]$ laminate in Figure 2 confirm the different postcure shapes of the laminates.

For the other laminates not classified as simple crossply laminates, the laminate will still snap through to another shape. After the force or load applied for snap through is removed, the laminate will return to its original shape, shape I, whereas for the simple cross-ply laminates, after removal of the snap through force, the laminate will continue to stay in the equilibrium cylindrical shape II. Therefore, due to instability, the [0/45] laminate will deform after actuation but will not stay in that shape. In Figure 3, the meshes for the room temperature shape and actuated displacement of the [0/45] laminate are shown.

\section{Numerical Models for Morphing Structures}

This research uses a multiphysics numerical software which was used to compare to analytical modeling and experimental results. The present models required implementing the Thermal-Structural Interaction mode. This mode couples both the structural mechanics and the heat transfer modules. The analysis types of the stress-strain and the heat transfer modules were chosen as static and transient types respectively. Due to the small thicknesses of the laminates studied, models were done using linear and quadratic mesh element types. The linear models were used initially for quicker solutions.

3.1. Initial Setup. The Thermal-Structural Interaction mode allowed for the inclusion of thermal expansion due to the coefficients of thermal expansion (CTE) during the cure process. As mentioned earlier, the CTEs along with geometry and other material parameters play key roles during the heating and cooling of the laminate. With the 3D space, new coordinate systems are needed to specify certain fiber orientations for plies not oriented in the global or principal material axes. This feature is done by rotating the global coordinate system from the original $x$-, $y$-, $z$-axes to the angle needed for the specific ply. Allowing $0^{\circ}$ to represent the default or global coordinate system (no need to change coordinate system if ply is oriented at $0^{\circ}$ ), the remaining plies coordinate systems can be changed using the consecutive rotation axes option. 


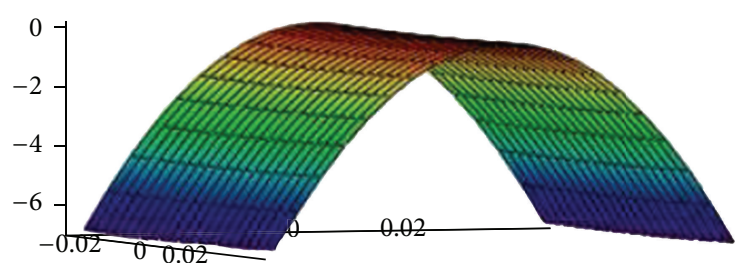

(a) Room temperature shape

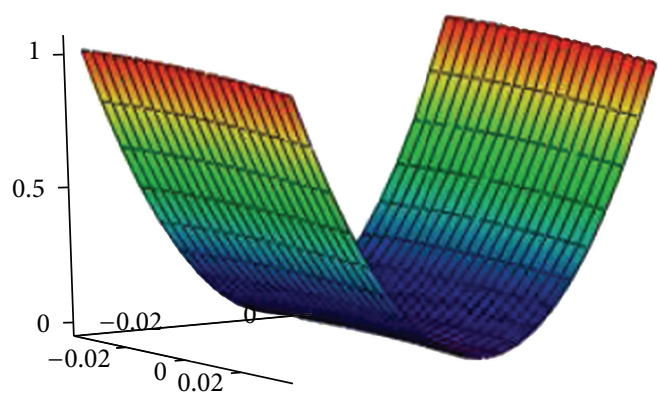

(b) Actuated shape

FIGURE 2: $\left[0_{2} / 90_{2}\right]$ room temperature shape versus actuated shape.

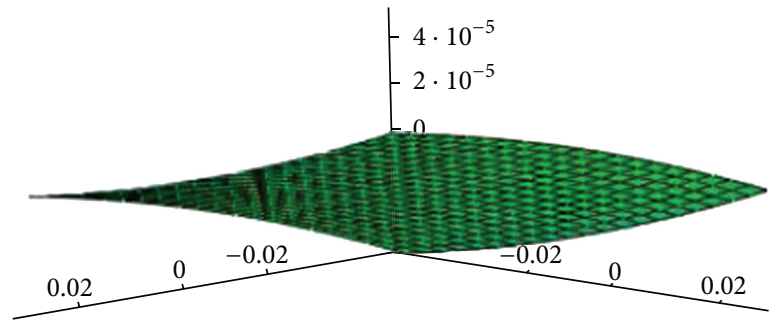

(a) Room temperature shape

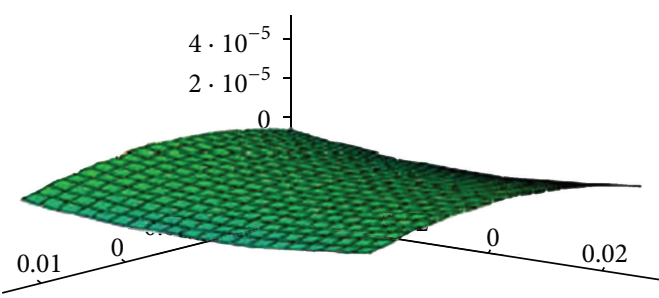

(b) Actuated shape

FIGURE 3: [0/45] room temperature shape versus actuated shape.

The laminate model was chosen to be treated as an orthotropic material. To model the mold press, the initial pressure of the laminate can be selected. Modeling free thermal expansion, there were no constraints on the boundaries or edges of the laminate. Compared with the analytical model, this assumption can be made due to the absence of external loads during the cooling of the laminate to room temperature. When solving the model, the solver parameters are important for accuracy and solver convergence. For the structural application mode, the solver was chosen based on the static analysis nature of the problem. This analysis type differs from the heat transfer application mode. For the heat transfer application mode, the analysis was chosen to be transient based on the steps of the curing process. Therefore, this analysis type is needed to couple both the static and transient nature of the curing process.

The numerical analysis allowed for more complex lamina behavior than the CLT assumptions used in the analytical modeling. For the structural mechanic analysis mode of the numerical modeling, a few important equations were used to determine the domain of the laminate. Using the solid, stressstrain application for 3D modeling, the strain-displacement relationship equations were specified [11]. The stress-strain relationship of the numerical analysis is the general Hooke's law formulation. The structural mechanics mode is based on the weak formulation of equilibrium equations in the global stress components. For 3D models, the equilibrium equation is represented by

$$
-\nabla \sigma=F
$$

where $\sigma$ is the stress tensor and $F$ is the volume or body forces [11]. The subdomain setting for the heat transfer mode was specified using Fourier's law:

$$
-\nabla(k \nabla T)=Q
$$

where $T$ is the temperature being measured at that instant, $Q$ is the heat source, and $k$ is the thermal conductivity. Specifying the heat transfer boundary equation using the heat flux equation,

$$
n(k \nabla T)=q_{o}+h\left(T_{\mathrm{inf}}-T\right)+\operatorname{Const}\left(T_{\mathrm{amb}}^{4}-T^{4}\right),
$$

where $q_{o}$ is the inward heat flux of the composite being modeled, $h$ is the heat transfer coefficient, $T_{\text {inf }}$ is the external temperature, Const is the numerical constant for heat transfer relations, and $T_{\mathrm{amb}}$ is the ambient temperature. The heat transfer boundary equation is a form of the heat convection equation. The second half of the heat transfer boundary equation is the heat transfer due to radiation. Although shown, it was not used in the models. The equations in this section are all derived or are a form of the same analytical equations used in the numerical analysis. 


\subsection{Actuation Morphing Models}

3.2.1. Analytical Models. For piezoelectric analysis, the plates or in this case MFC actuators were classified as thin plates. Thin plates are those with a ratio of thickness to length less than $1 / 20$. This allows the complex three-dimensional problem to be reduced to a problem in two dimensions [10]. Using the same assumptions of the classical plate theory, the thin plate is now classified as a plane stress problem. The analysis implements the total potential energy approach similar to the postcure shape analytical work. The Ritz method is used for the variational principal solution and is reduced to a set of linearly independent equations by an assumed form of generalized coordinates. The generalized coordinates are then used to represent the potential energy of the system. The strain vector is then given by the expression

$$
S(x)=L_{u} u(x)
$$

where $L_{u}$ is the differential operator and $u(x)$ are the displacements as a function of the generalized coordinates $r$ with $N_{r}(x)$ as the set of admissible (satisfying conditions) shape functions:

$$
u(x)=N_{r}(x) r .
$$

The relationship between the strain vector and generalized coordinates can now be made:

$$
S(x)=L_{u} N_{r}(x) r=B_{r}(x) r .
$$

With the assumptions of the thin plate and classical plate theory, the strain-displacement relationship for a thin plate can be written as follows:

$$
\left\{\begin{array}{l}
S_{1}(x) \\
S_{2}(x) \\
S_{3}(x) \\
S_{4}(x) \\
S_{5}(x) \\
S_{6}(x)
\end{array}\right\}=\left[\begin{array}{ccc}
\frac{\partial}{\partial x} & 0 & -z \frac{\partial^{2}}{\partial x^{2}} \\
0 & \frac{\partial}{\partial x} & -z \frac{\partial^{2}}{\partial x^{2}} \\
0 & 0 & 0 \\
0 & 0 & 0 \\
0 & 0 & 0 \\
\frac{\partial}{\partial y} & \frac{\partial}{\partial x} & -2 z \frac{\partial^{2}}{\partial x y}
\end{array}\right]\left\{\begin{array}{l}
u_{1}(x) \\
u_{2}(x) \\
u_{3}(x)
\end{array}\right\} .
$$

Due to the parameters of the MFC actuator operating in $d_{33}$ mode, the deflection will only be in the transverse direction. This simplifies (17) to only the transverse displacement in the third direction, $u_{3}(x)$. The stiffness matrix for the substrate and the piezoelectric material can then be determined. The coupling matrix, which consists of the coupling coefficient for the piezoelectric material, is then found by integration through the length and placement of the MFC actuator:

$$
\Theta_{i}=\frac{2 t_{s} t_{p}+t_{p}^{2}}{8 a_{p} b_{p}} \int_{x_{p}}^{x_{p}+a_{p}} \int_{y_{p}}^{y_{p}+b_{p}}\left(h_{13} \frac{\partial^{2} \phi_{i}}{\partial x^{2}}+h_{23} \frac{\partial^{2} \phi_{i}}{\partial y^{2}}\right) d y d x,
$$

where $t_{s}$ and $t_{p}$ are the thickness of the actuator and the substrate, $h_{i j}$ is the coupling coefficients, and $a_{p}, b_{p}, x_{p}, y_{p}$ are
TABLE 1: Material properties for MFC P1 $d_{33}$ actuator.

\begin{tabular}{lcc}
\hline Mechanical & Electrical & Coupling \\
\hline$d_{33}=460 \mathrm{pm} / \mathrm{V}$ & $\beta_{33}^{S}=.42 \mathrm{nF} / \mathrm{cm}^{2}$ & $h_{13}=400 \mathrm{pC} / \mathrm{N}$ \\
$d_{31}=-210 \mathrm{pm} / \mathrm{V}$ & & $h_{23}=-170 \mathrm{pC} / \mathrm{N}$ \\
$E_{1}=30.336 \mathrm{GPa}$ & & \\
$E_{2}=15.857 \mathrm{GPa}$ & & \\
$v_{12}=.31$ & & \\
$v_{21}=.16$ & & \\
$G_{12}=5.515 \mathrm{GPa}$ & & \\
\hline
\end{tabular}

the lengths, and placements of the actuator. The capacitance matrix is then computed by

$$
C_{p}^{S}=\left[\begin{array}{cc}
\frac{1}{2} \frac{\beta_{33}^{S} t_{p}}{a_{p} b_{p}} & 0 \\
0 & \frac{1}{2} \frac{\beta_{33}^{S} t_{p}}{a_{p} b_{p}}
\end{array}\right] .
$$

With the material properties shown in Table 1 , the deflection field $u_{3}$ for the piezoelectric material system can be found.

After the curing process is simulated, the same models can be used to model the piezoelectric actuation. Running the structural mechanics and heat transfer modules together gave the postcure room temperature shapes of the cross-ply laminates. Using these existing models, the 3D piezoelectric solid module was added. This allowed for the same postcure curvature (same geometries from cure process models) to be used for modeling piezoelectric actuation. The same element types were used as in the fabrication models.

The work in the previous section had to be done to account for the cooled room temperature shapes of the composite laminates. After the curing process is simulated, the same models can be used to model the piezoelectric actuation by adding the $3 \mathrm{D}$ piezoelectric solid module. This allowed for the same postcure curvature to be used for modeling the piezoelectric actuation. After drawing the rectangular $27.9 \times$ $13.995 \mathrm{~mm}$ MFC actuator at the center of the $76.2 \mathrm{~mm}^{2}$ square and $76.2 \times 127 \mathrm{~mm}^{2}$ rectangular laminate on the first ply, the laminate and actuator were bonded to the laminate by creating a contact pair between the bottom surface of the MFC actuator and the top surface of the laminate. This was done to make the MFC actuator and laminate one domain. For the numerical models, the MFC actuator was bonded to the laminate according to the direction of the first ply.

Using the same structural mechanics domain settings from the cure process models, the ply orientation, thickness, and material are recalled for the 3D piezoelectric solid module. This research implements the converse piezoelectric effect which is characterized by applying a force (voltage in this case) to a piezoelectric solid. The numerical models used piezoelectric equations in the stress-strain form. The basic 


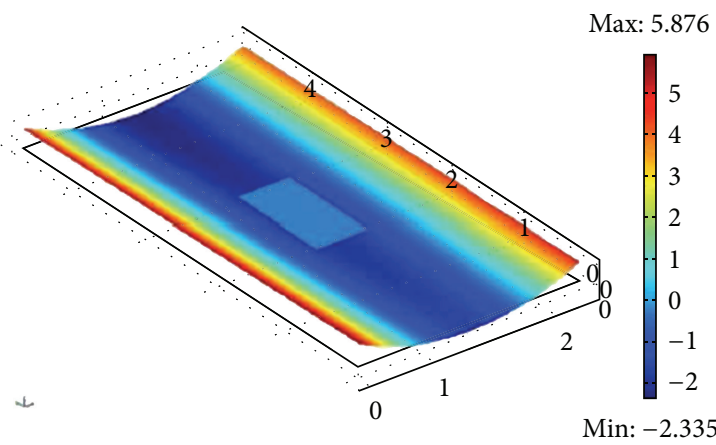

(a)

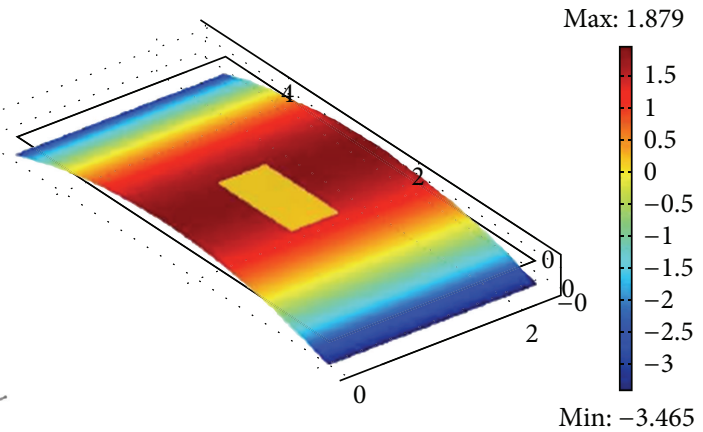

(b)

Figure 4: Rectangular [0/90] laminate cylindrical shapes I and II.

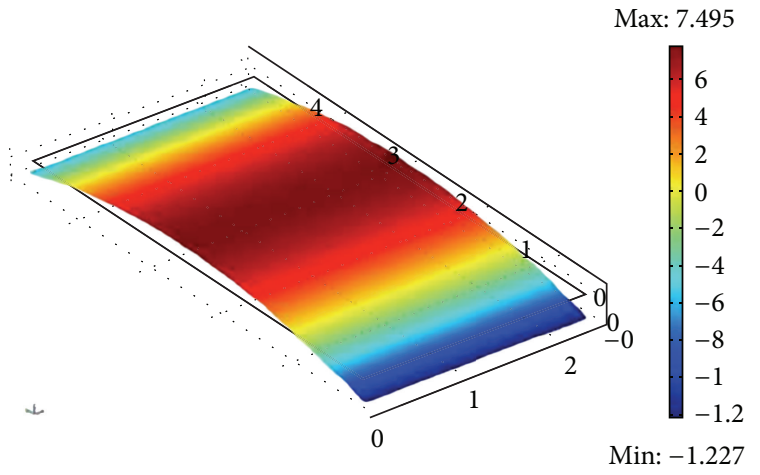

(a)

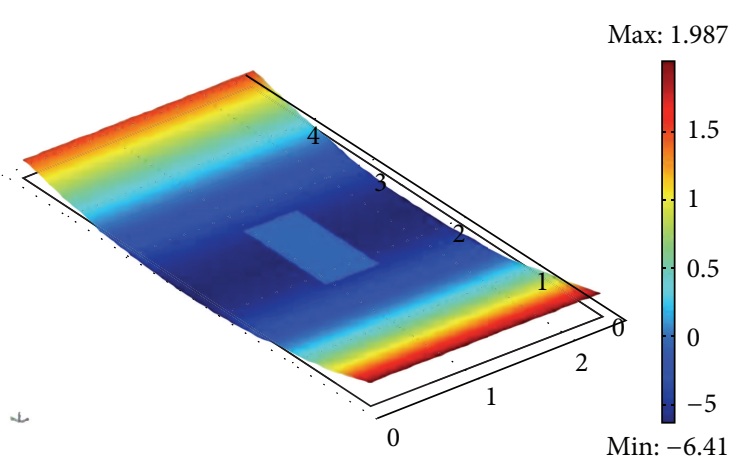

(b)

FIGURE 5: Rectangular $\left[0_{2} / 90_{2}\right]$ laminate cylindrical shapes I and II.

constitutive equations for the piezoelectric coupling effect for stress and electric displacement are as follows:

$$
\begin{gathered}
T=c_{E} S-e^{T} E, \\
D=e S+\varepsilon_{S} E,
\end{gathered}
$$

where the variables $T, c, E, S, e, D, \varepsilon_{s}$ are the stress, Young modulus, electric field, strain, piezoelectric constant, electric displacement, and the permittivity of the piezoelectric material. There are three material models for domain definition when dealing with the piezoelectric application mode and they are piezoelectric, decoupled, isotropic, and decoupled, anisotropic [11]. The decoupled, anisotropic model was used for the actuation models. Using this modeling, the full six-bysix elasticity matrix was defined. After the material properties of the MFC actuator are defined, the electric boundary conditions are then determined. For voltage application, the electrostatics formulation for static decoupled, anisotropic is solved:

$$
-\nabla\left(\varepsilon_{0} \varepsilon_{r} \nabla V\right)=\rho_{v}
$$

where $\varepsilon_{0}, \varepsilon_{r}, V$, and $\rho_{v}$ are the electrical permittivity of free space, the relative electrical permittivity, voltage, and volume charge density. The other sides of the MFC actuator were classified as the zero charge/symmetry sides where

$$
n D=0
$$

specifies that the normal component of the electric displacement is zero. This is used for the rest of the sides to keep symmetry with respect to the boundaries. Using the numerical models as predictions of experimentations, a high input voltage was used to observe deformation in the model. Applying an initial $1000 \mathrm{~V}$ to the actuator, magnitude will change based on the experimental snap through, and the snap through to the orthogonal cylindrical shape (cylindrical shape II) was observed. Figure 4 shows both the postcured shape and the actuated shape (cylindrical shapes I and II) of the $[0 / 90]$ rectangular laminate.

The transverse displacement in the actuated laminate is seen Figure 4(b). The postcure curvature in Figure 4(a) is seen in the negative $z$-direction along the $x$-axis. The expected actuated displacement is to be orthogonal to the cylindrical shape I. This displacement can be seen in Figure 4(b) where the transverse displacement is now in the positive $z$-direction along the $y$-axis for the rectangular [0/90] laminate. The same was observed with the square [0/90] laminate. Modeling the rectangular and 


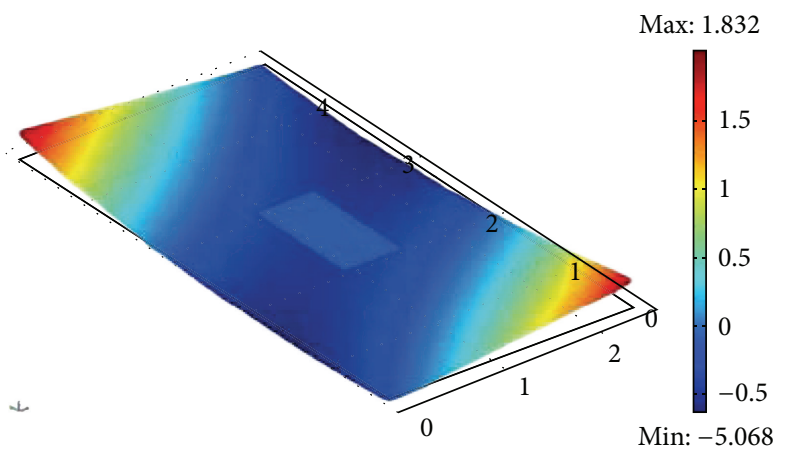

(a)

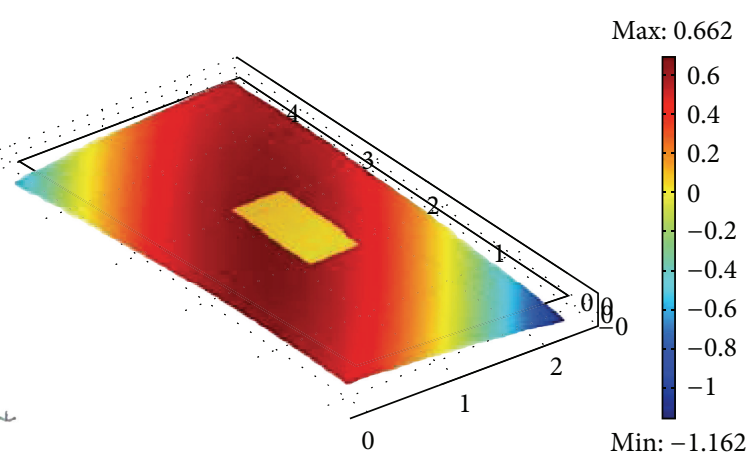

(b)

FIGURE 6: Rectangular [0/45] laminate postcure and actuated shapes.

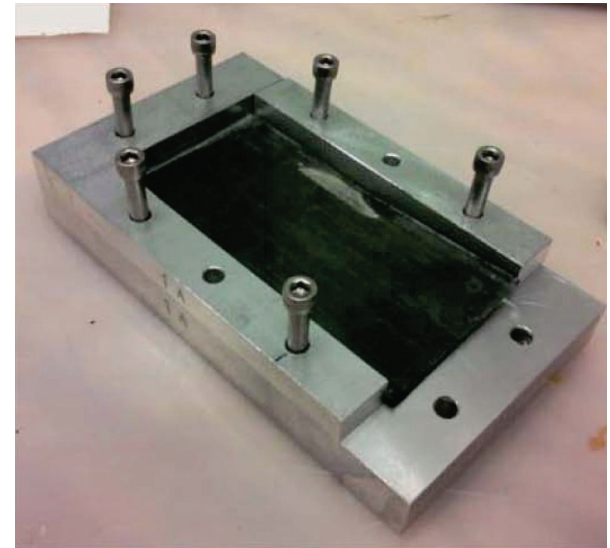

(a)

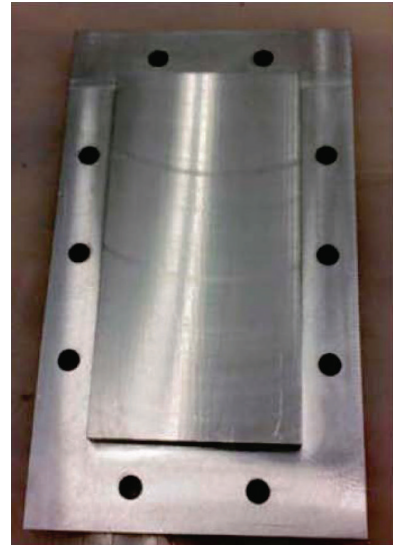

(b)

FIGURE 7: Mold for fabrication.

square $\left[0_{2} / 90_{2}\right]$ laminates after the cure process, the deformation shape observed was in the opposite direction of the [0/90] laminate. Therefore, after actuation, the cylindrical shape II of the $\left[0_{2} / 90_{2}\right]$ laminate should look similar to the [0/90] postcure shape since that is the cylindrical shape orthogonal to cylindrical shape I.

Seen in Figure 5, the postcure shape and actuated displacement of the rectangular $\left[0_{2} / 90_{2}\right]$ laminate are cylindrically orthogonal to each other. This actuated displacement is expected in the experimentation. For the rectangular $\left[0_{2} / 90_{2}\right]$ laminate, the postcure shape deformation is in the positive $z$ direction along the $y$-axis. When the voltage is applied to the MFC actuator, the $\left[0_{2} / 90_{2}\right]$ model snaps through to cylindrical shape II. Cylindrical shape II is orthogonal to the postcure deformation shape, cylindrical shape I, as expected. The same observations were made with the square $\left[0_{2} / 90_{2}\right]$ model. For any laminate not classified as a thin simple cross-ply laminate, the postcure deformation shape will be that of the saddle shape. Applying a voltage to the MFC actuator will cause deformation in the laminate, but once the voltage load (actuation magnitude) is lowered or removed, the laminate will return to its original shape, the postcure deformation shape.
Modeling the [0/45] laminate with these expectations in mind, the actuation model was done with a static solver to show the unstable shape while the voltage load was still applied. In Figure 6 both shapes are shown.

\section{Experimental Results}

4.1. Fabrication. Using AS4 carbon fiber prepregs, the unsymmetric laminates were fabricated. A standard hand layup procedure was used to stack the plies of the laminate. Both square and rectangular laminates were fabricated to better understand the role of the material properties and geometry in piezoelectric actuation. A mold was designed to press the laminate flat while heating to the cure temperature. A convection oven at Raspet Flight Research Laboratory was used for curing the composite laminates. The mold seen in Figure 7 held the composite laminate flat with a pressure of $5.861 e^{5} \mathrm{~Pa}$ while in the oven. At a cure temperature of $449.817 \mathrm{~K}$, the laminate is held flat under the press/mold at this temperature for three and a half hours.

The mold and press are then removed, and the unsymmetric laminate now exhibits the ability to deform to 


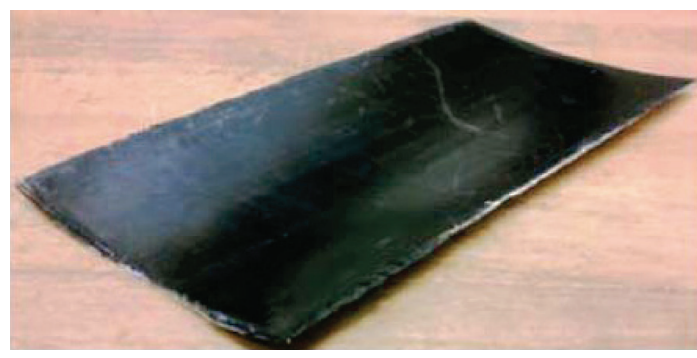

(a)

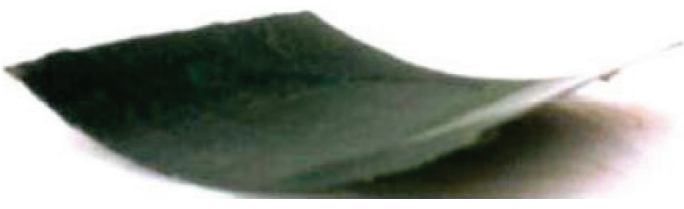

(b)

FIGURE 8: Rectangular and square cured room temperature [0/90] laminates.

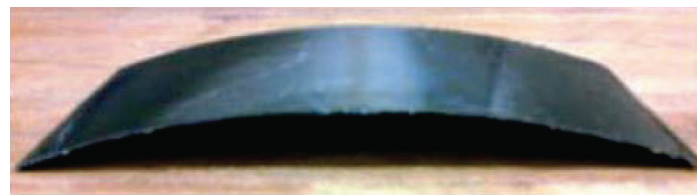

(a)

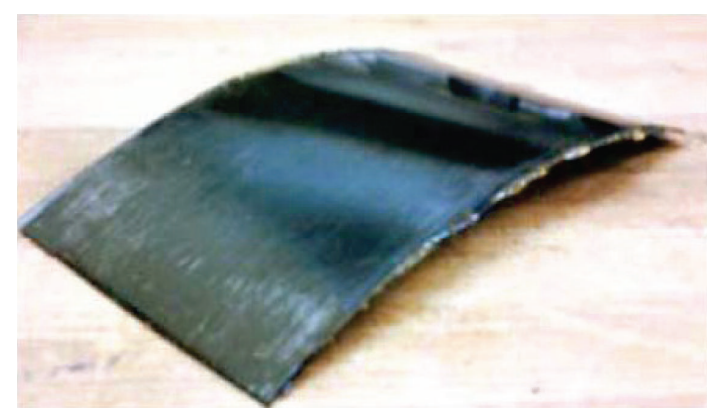

(b)

FIgURE 9: Rectangular and square cured room temperature $\left[0_{2} / 90_{2}\right]$ laminates.

a postcure shape. Unsymmetric laminates and the snap through phenomenon work best when the laminate can be classified as a thin laminate. Thin laminates, where the length and width are large compared to the thickness, will deform to one of two circular cylindrical shapes as discussed earlier [11]. After removal of the mold from the oven, the press is released allowing the laminate to deform freely. The laminate is then left over night to complete cooling. With the analytical and numerical results, the laminates are expected to deform to room temperature postcure shapes discussed in earlier sections. Figure 8 shows both geometries of the [0/90] laminate after the cure process. The cylindrical curvature seen in Figure 8 for the [0/90] laminate is similar to the prediction of the analytical and numerical models.

The next composite fabricated was the $\left[0_{2} / 90_{2}\right]$ laminate. As the analytical and numerical models suggest, the curvature is expected to be in the positive $z$-direction, opposite that of the postcure shape of the [0/90] laminate.

The postcure transverse curvature in the $\left[0_{2} / 90_{2}\right]$ is greater than the curvature of the [0/90] laminate. This is possible due to the increase of the number of plies in the laminate. Although the transverse curvature is greater, with more plies the laminate becomes stiffer, therefore, making it more difficult to apply force for snap through without damaging the laminate. The next composite fabricated was the [0/45] laminate. This laminate, as discussed earlier, is expected to deform to a saddle shape. The twist curvature that arose from the cooling of the laminate is seen in both the square and rectangular laminate in Figure 10. The curvature is similar to numerical models of the [0/45] laminate.

For comparisons seen in later chapters, the transverse displacement of the deformed laminates were measured. Due to the symmetric curvature of the simple cross-ply laminates, $\left[0_{n} / 90_{n}\right]$ where $n$ is the number of plies, this symmetric cylindrical shape can be measured using a FARO gage coordinate-measuring machine (CMM). The CMM establishes a coordinate system for the laminate. This coordinate system is then used to measure the deformation and mesh the postcure room temperature laminate. The meshes for the rectangular $[0 / 90]$ and $\left[0_{2} / 90_{2}\right]$ laminates are seen in Figure 11 .

For the simple cross-ply laminates, meshes were made with the FARO gage CMM to compare the transverse displacement due to the cure process. There were key components for the fabrication process to insure postcure deformation. The thin composite laminates have to be held flat during the heating part of the cure process. After the elevated cure temperature is held for three and a half hours, the laminate is removed from the oven and cools to room temperature. During this cooling process, the press from the mold is removed allowing the laminate to deform freely. Analytical and numerical models were used as predictions of the 


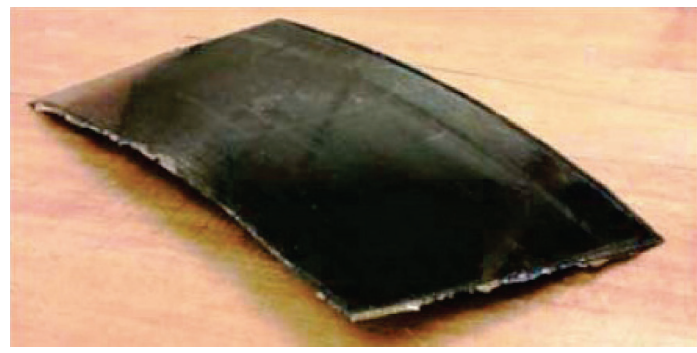

(a)

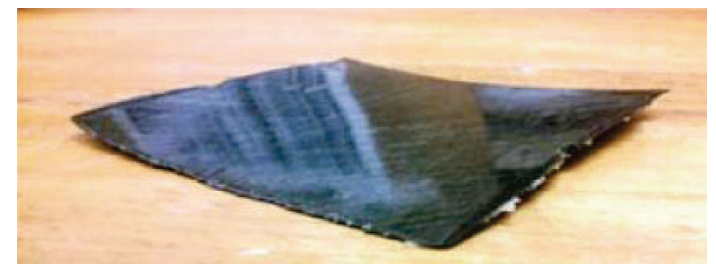

(b)

FIGURE 10: Rectangular and square cured room temperature [0/45] laminates.

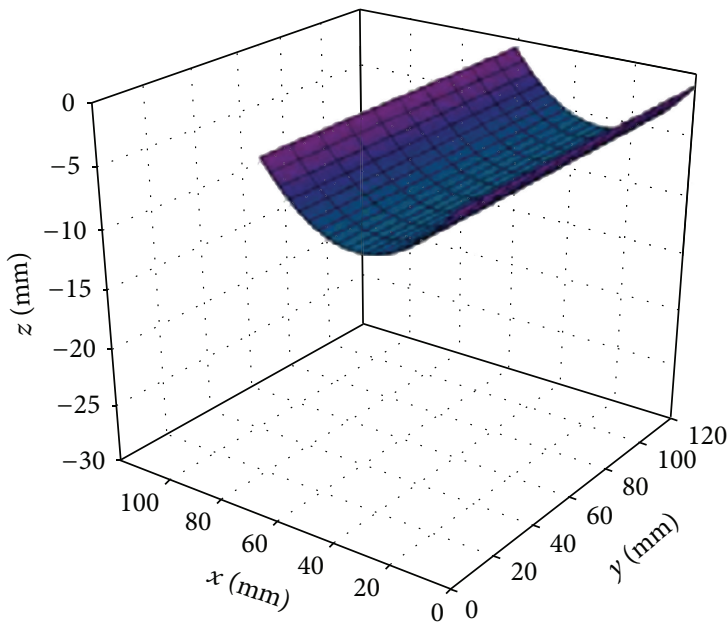

(a)

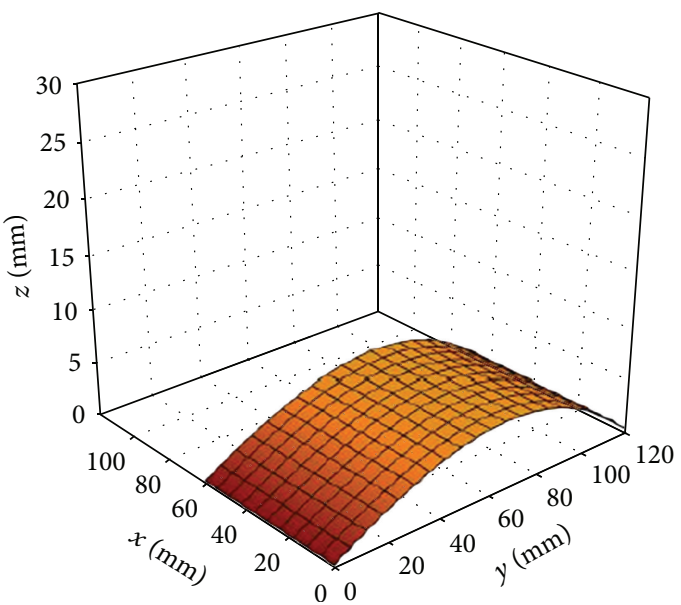

(b)

FIGURE 11: Rectangular mesh of $[0 / 90]$ and $\left[0_{2} / 90_{2}\right]$ laminates.

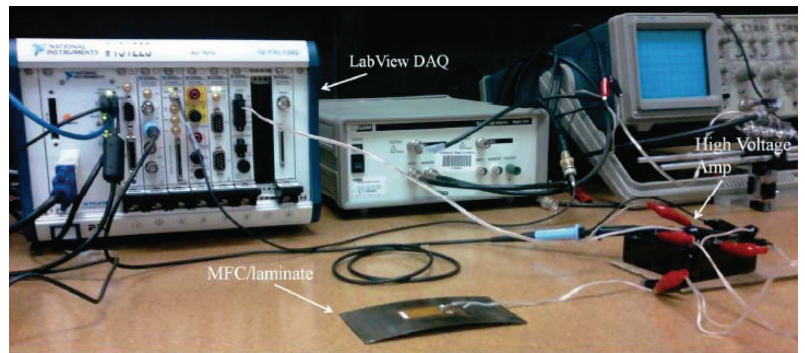

FIGURE 12: Experimental setup.

postcure shapes of the laminates. The fabrication process was heavily investigated due to the effects that it has on piezoelectric morphing modeling. The fabrication models were used to more accurately model piezoelectric morphing structures. These models can now be used as preliminary models for piezoelectric actuation.

After cooling the composite laminates, the MFC actuator from Smart Material Corp. can be bonded to the laminate. The bonding process done in this research was the process suggested by the Smart Material Corp. The epoxy was applied to the bottom surface of the MFC actuator then attached to the deformed postcure shape laminate. The bond is then clamped together over night to secure adhesion. The experimental setup is shown in Figure 12.

Using the LabView data acquisition system, input and output voltages were supplied and monitored. The first stacking sequence experimented with was the [0/90] rectangular and square laminate. For experimentation, the programming voltage was increased from zero by $.02 \mathrm{~V}$ DC per 45 seconds until snap through was observed. After snap through of the laminate to the orthogonal cylindrical shape II, the voltage load is removed to assure the cylindrical shape II is stable. If the shape was unstable, removing the load (voltage) would cause the laminate to snap back to cylindrical shape I as discussed earlier. With the process described above, piezoelectric actuation was done with the rectangular [0/90] laminate. In Figure 8, the transverse actuated displacement is shown in the second picture.

After running the experimentation process a number of times and experiencing snap through, it was observed that for the rectangular [0/90] laminate an estimated voltage at snap through was 391 V DC. As seen in Figures 4 and 13, the postcure deformation shape and the piezoelectric actuated shape are similar. Therefore, the numerical model proved as a reasonable prediction for the actuated displacement of 


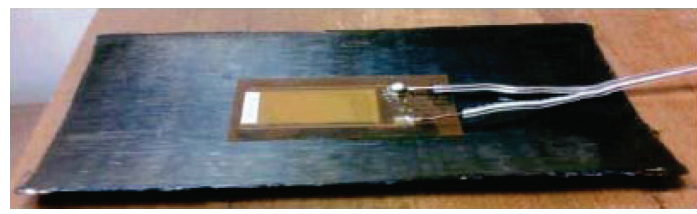

(a) Cylindrical shape I

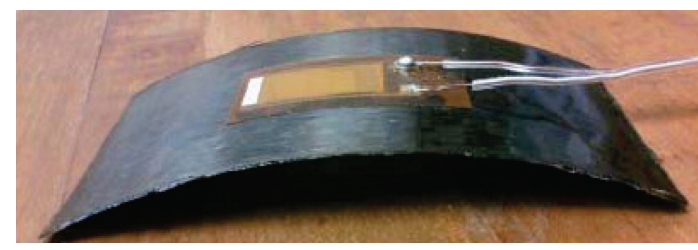

(b) Cylindrical shape II

Figure 13: Cylindrical shapes I and II of the rectangular [0/90] laminate.

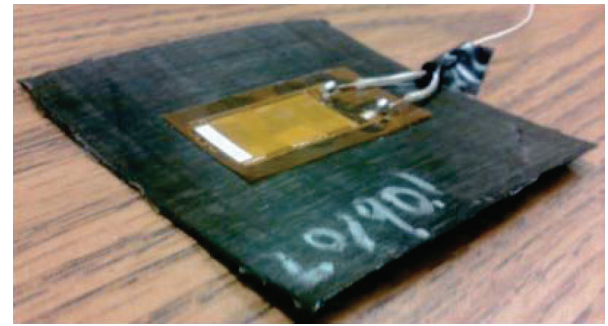

(a) Cylindrical shape I

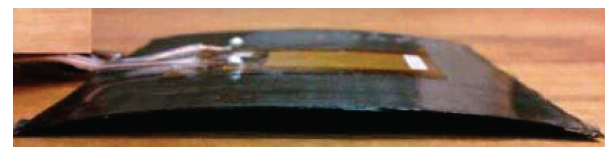

(b) Cylindrical shape II

FIGURE 14: Cylindrical shapes I and II of the square [0/90] laminate.

the rectangular [0/90] laminate. This voltage magnitude was then used in the numerical model to get better results for comparisons. The same procedure was done with the square [0/90] laminate, seen in Figure 14. The estimated voltage at snap through was $951.45 \mathrm{~V} \mathrm{DC}$, which was close to three times more than the snap through voltage for the rectangular laminate. The smaller surface area of the square laminate increased the stiffness, thereby increasing the snap-through voltage.

The same procedure was done with the square [0/90] laminate, seen in Figure 9. The estimated voltage at snap through was $951.45 \mathrm{~V} \mathrm{DC}$, which was close to three times more than the snap through voltage for the rectangular laminate.

Showing similar actuated shapes as the analytical model suggested, the square and rectangular cross-ply laminates behaved as expected. This was done by using the preliminary methods and techniques for modeling the cure process. With the results from those preliminary models, the piezoelectric actuation effect can be more accurately modeled. The next stacking sequence for piezoelectric experimentation was the rectangular and square $\left[0_{2} / 90_{2}\right]$ laminate. With more plies in the laminate, the expected bending stiffness is going to be more than that of the [0/90] laminate. Referring back to the bending stiffness equation,

$$
D_{i j}=\sum_{k=1}^{n}\left(E_{i j}\right)_{k}\left(\frac{z_{k}^{3}-z_{k-1}^{3}}{3}\right)
$$

the thicker laminate will have higher magnitudes in the bending stiffness matrix. Although thicker, the $\left[0_{2} / 90_{2}\right]$ laminate can be classified as a thin laminate due to the ratio of the thickness to length being less than 1/20. After multiple fabrication attempts with the AS4 carbon fiber, it was noticed that the stiffness of the $\left[0_{2} / 90_{2}\right]$ laminate might be too dominating for the MFC actuator to snap through. The MFC actuator has a limited maximum force of free strain. After many attempts of increasing and holding the programming voltage up to $1500 \mathrm{~V} \mathrm{DC}$, which is the maximum input, the laminate did not snap through due to the increased stiffness of the laminate. With an actuator that can produce a higher force or displacement (free strain), the snap through could occur. With the knowledge of the saddle shape and its instability, experiments were done with the other laminates not classified as the simple cross-ply laminates. The rectangular and square $[0 / 45]$ laminates were experimented with piezoelectric actuation to confirm the instability issues arising from the ply orientation. Test done to observe if these laminates will snap back to their original shape. The same procedure was done as with the simple cross-ply laminates. Due to the nature of the saddle shape, the [0/45] laminate was modeled analytically and numerically.

\section{Morphing Comparisons}

As seen in previous sections for the [0/90] laminate, the snap through from cylindrical shape I should cause the laminate to have actuated displacement along the $y$-axis in the positive $z$-direction, this being cylindrical shape II. The rectangular [0/90] laminate is shown in Figure 15.

As observed in earlier chapters, the actuated displacement curvature is predicted by the analytical and numerical models. These predictions are then compared to the experimental results. The rectangular [0/90] laminate comparisons of the numerical and experimental results are reasonably accurate. As expected, the actuated snap through curvature of the rectangular [0/90] laminate is along the $y$-axis, which is the orthogonal cylindrical shape II. This proves that the numerical and analytical models are reasonable prediction models for actuation. The same actuated snap through curvature is expected for the square [0/90] laminate shown in Figure 16.

It can be seen that with the same actuation voltage used, the numerical model for the rectangular [0/90] laminate are reasonably close to the experimental result. For the square $[0 / 90]$ laminate, the error in the comparison for the actuated displacement is more evident here. The numerical model 


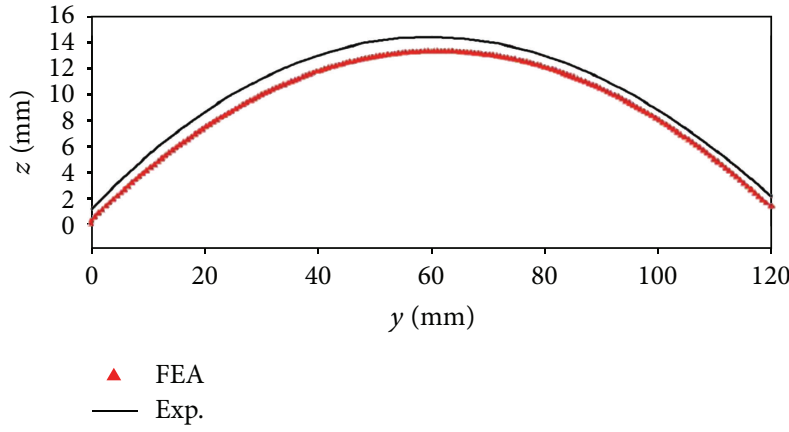

(a)

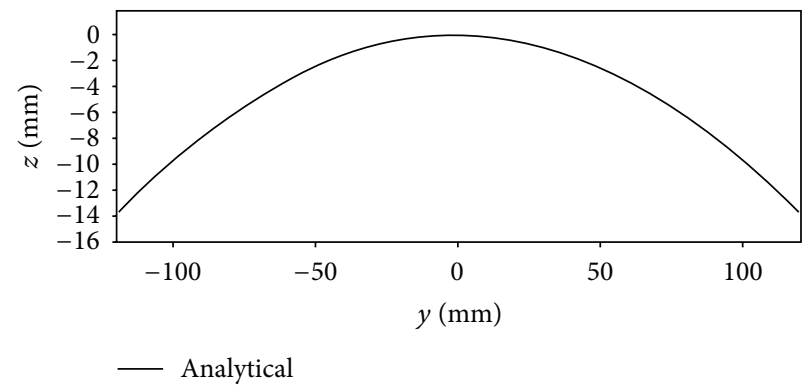

(b)

FIGURE 15: Rectangular [0/90] laminate actuated displacement.

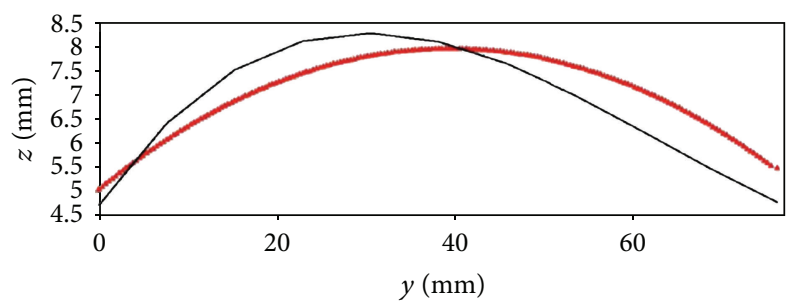

$\triangle$ FEA

- Exp.

(a)

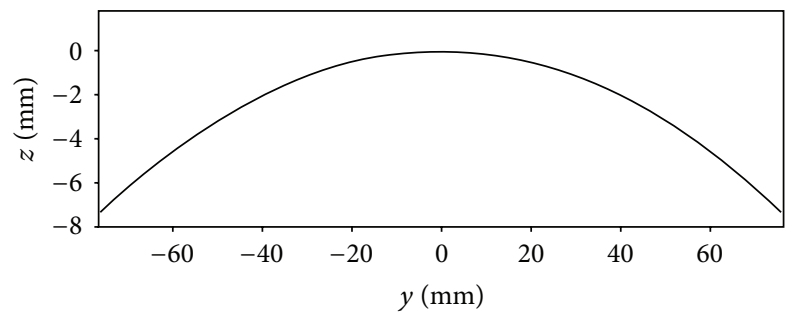

(b)

Figure 16: Square [0/90] laminate actuated displacement.

of the square [0/90] laminate exhibits a more symmetric curvature of the actuated displacement. The numerical model consists of ideal parameters. When conducting the experimentation for the cross-ply laminates, the situations were not as ideal. Due to the size and flexibility of the laminate, when using the FARO gage coordinate measure machine (CMM) to obtain data points, the laminate will tend to move. The data obtained from the experimental actuation therefore will not be as precise as the numerical ideal models. When comparing the actual experimental result to the numerical model, this error can be seen. Table 2 represents the maximum snap through displacement of the laminates. The actuated displacement is measured as a new shape; therefore, the measurements shown are not the displacement difference between cylindrical shapes I and II. For the thicker $\left[0_{2} / 90_{2}\right]$ laminates,
TABle 2: Actuated snap through displacement (mm).

\begin{tabular}{lcc}
\hline & $\begin{array}{c}{[0 / 90] \text { rectangular snap }} \\
\text { through voltage @ 391 } \\
\text { VDC }\end{array}$ & $\begin{array}{c}\text { [0/90] rectangular snap } \\
\text { through voltage @ 953.45 } \\
\text { VDC }\end{array}$ \\
\hline Experiment & 13.9718 & 8.5377 \\
Numerical & 13.121 & 7.893 \\
Analytical & 13.825 & 7.435 \\
\hline
\end{tabular}

piezoelectric actuation was done analytically and numerically. Due to the thickness and increased stiffness of the $\left[0_{2} / 90_{2}\right]$, these laminates were not stable and could not snap through. The laminates not classified as simple cross-ply laminates were investigated analytically, numerically, and experimentally. For piezoelectric actuation, test was done on these laminates to assure that the laminates were unstable and that they would snap back to their original postcure shape after the actuation voltage is removed.

\section{Conclusions}

Piezoelectric actuation was investigated analytically, numerically, and experimentally. Analytically, actuation was modeled similarly to cure process models. The sum of the total potential energies was used to characterize the actuation of the thin laminates. One "1" represented the total potential energy when the "driving force" was the heat transfer occurring in the laminate. Two " 2 " represented the total potential energy when the "driving force" was the stresses and strains of the MFC actuator. This was necessary to account for the deformation of the thin laminate after the cure process. Together, these equations gave reasonable predictions of the laminates snapping through to cylindrical shape II. Numerical models were implemented next to observe similar results. This was done by using the results from the same heat transfer modules used in the fabrication analysis. These results were then coupled with structural and piezoelectric modules. The numerical models for piezoelectric actuation were similar to the predictions given by the analytical models. Experimentation was done next with an MFC actuator from Smart Material Corp. The MFC actuator was bonded to the 
deformed thin laminates and clamped over night to assure bondage. Test was run by applying a voltage to the MFC actuator. Voltage loads were made by increments of .02 V DC from an input of 0 magnitude. This was done until snap through to cylindrical shape II was observed. The voltage load was then removed to test the stability of the laminates. For comparisons, after fabrication and for piezoelectric actuation, the transverse displacement of the composite laminates were measured using a FARO gage CMM. It was observed that the simple cross-ply laminates deformed to a symmetric cylindrical room temperature shape. As for the laminates not classified as cross-ply laminates, they deformed to room temperature shapes of the saddle form. The curvature of the saddle shape was not symmetric as the cross-ply laminates. For the simple cross-ply laminates that were stable, snap through to cylindrical shape II was accomplished. The actuated displacement was then measured with the FARO gage CMM and then compared to the analytical and numerical models. Although the CMM caused a few errors, the comparisons proved that the models were reasonably predicting the snap through displacement of the laminates. For the laminates not classified as cross-ply laminates, experimentation was done to assure that upon removal of the snap through voltage to shape II, the laminate would return to its original shape.

\section{References}

[1] R. M. Jones, Mechanics of Composite Materials, Taylor and Francis Group, New York, NY, USA, 2nd edition, 1999.

[2] M. R. Wisnom, M. Gigliotti, N. Ersoy, M. Campbell, and K. D. Potter, "Mechanisms generating residual stresses and distortion during manufacture of polymer-matrix composite structures," Composites A, vol. 37, no. 4, pp. 522-529, 2006.

[3] M. W. Hyer, "Calculations of the room temperature shapes of unsymmetric laminates," Journal of Composite Materials, vol. 15, pp. 296-310, 1981.

[4] M. L. Dano and M. W. Hyer, "Snap-through of unsymmetric fiber-reinforced composite laminates," International Journal of Solids and Structures, vol. 39, no. 1, pp. 175-198, 2001.

[5] M. L. Dano and M. W. Hyer, "Thermally-induced deformation behavior of unsymmetric laminates," International Journal of Solids and Structures, vol. 35, no. 17, pp. 2101-2120, 1998.

[6] M. Schlecht, K. Schulte, and M. W. Hyer, "Advanced calculation of the room-temperature shapes of thin unsymmetric composite laminates," Composite Structures, vol. 32, no. 1-4, pp. 627-633, 1995.

[7] M. R. Schultz, M. W. Hyer, R. Brett Williams, W. Keats Wilkie, and D. J. Inman, "Snap-through of unsymmetric laminates using piezocomposite actuators," Composites Science and Technology, vol. 66, no. 14, pp. 2442-2448, 2006.

[8] J. F. Nye, Physical Properties of Crystals: Their Representation By Tensors and Matrices, Oxford Science Publications, 1985.

[9] "MFC Brochure," 1990 Main Street, Suite 750, Sarasota, Fla, USA, 34236, 2010.

[10] D. J. Leo, Engineering Analysis of Smart Material Systems, John Wiley \& Sons, Hoboken, NJ, USA, 2007.

[11] COMSOL Multi-physics 3.5a, Structural Mechanics Module User's Guide, 2008. 

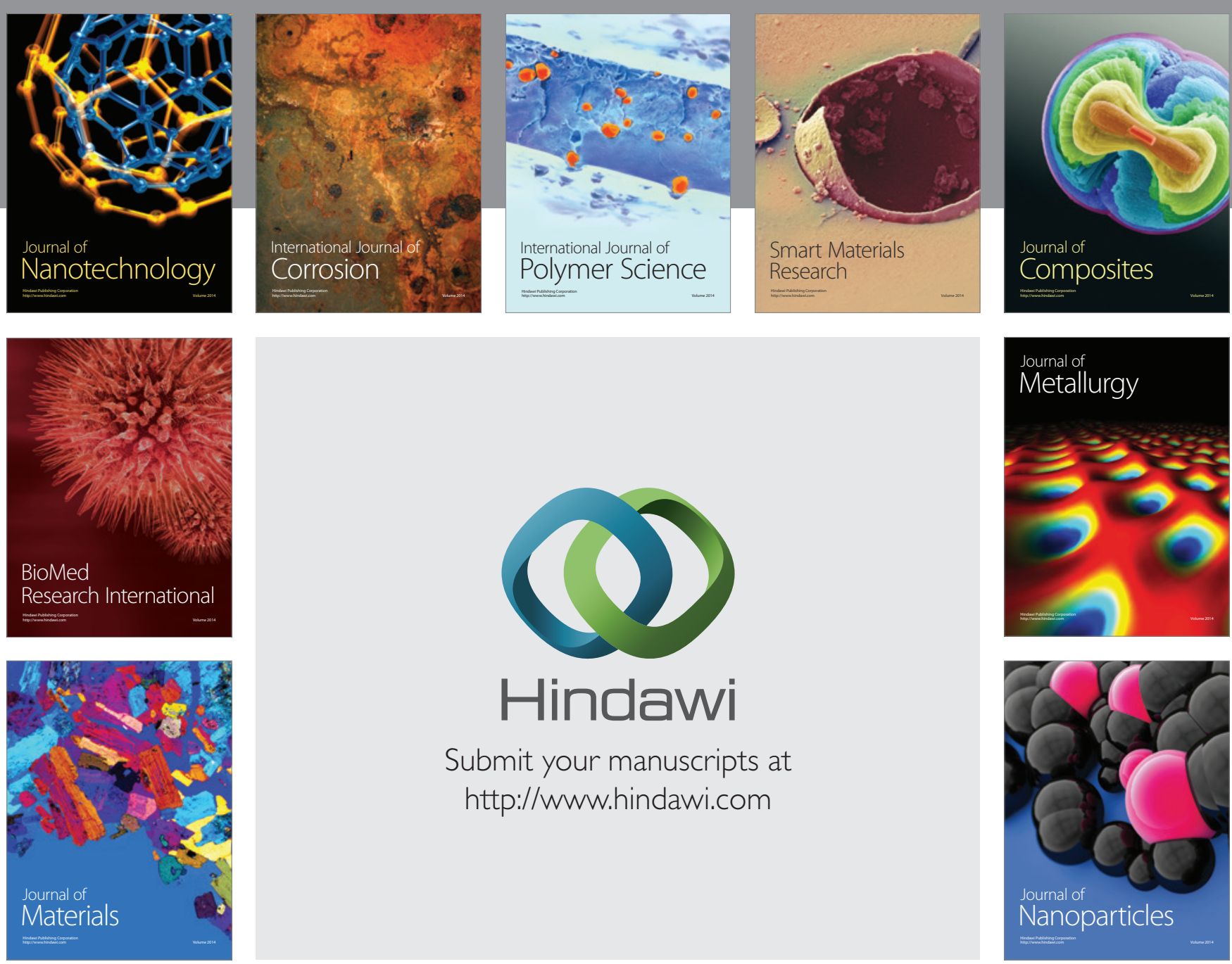

Submit your manuscripts at http://www.hindawi.com
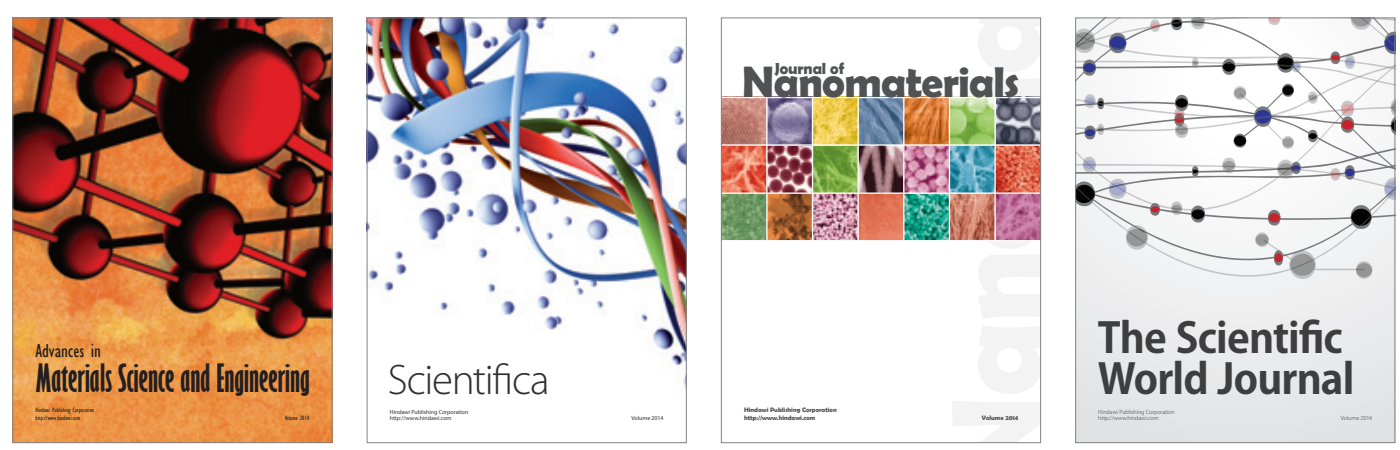

\section{The Scientific World Journal}
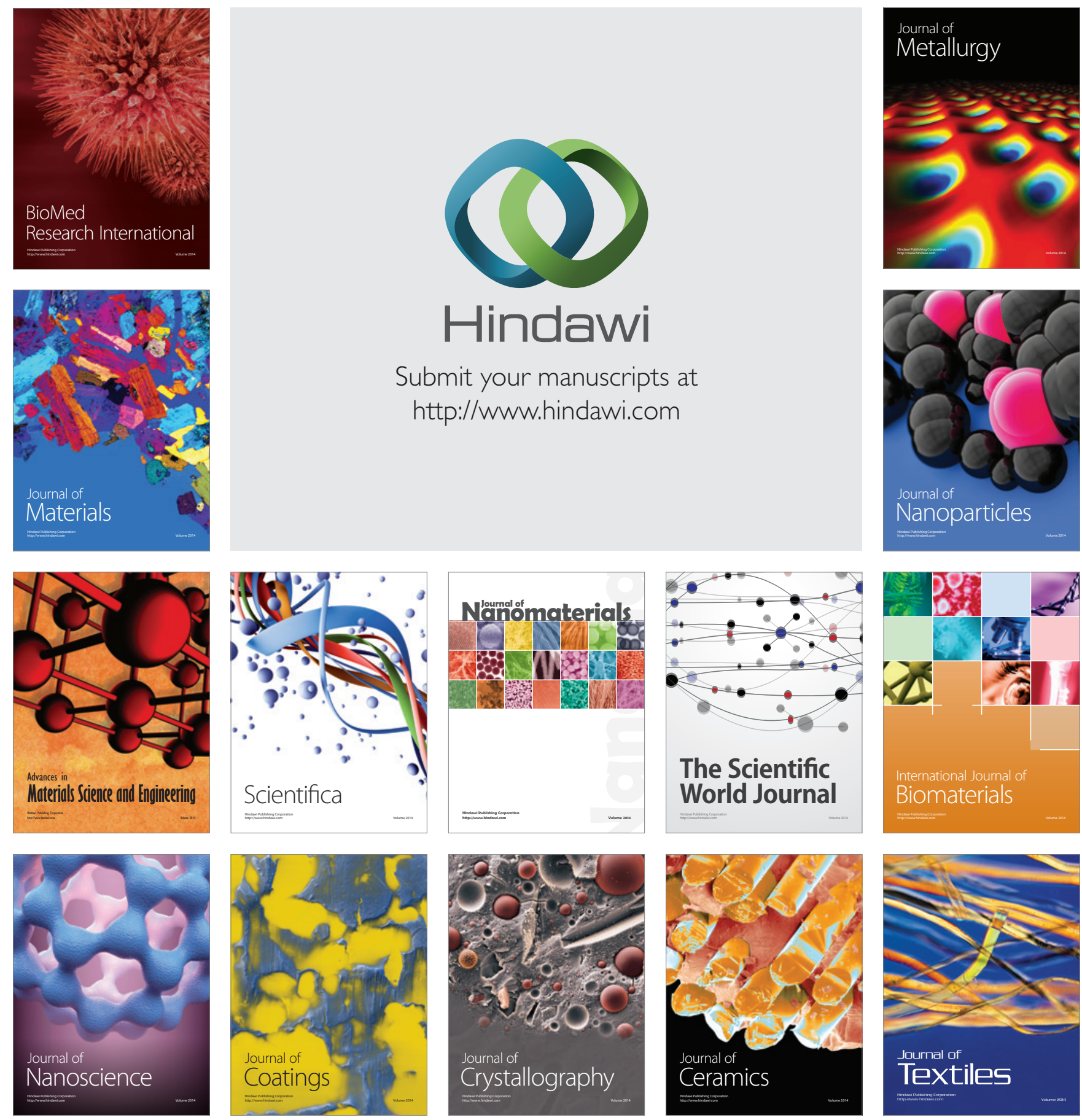